\title{
Genomic Features of Salmonella enterica Subspecies houtenae Serotype 45:g,z51:- Isolated from Multiple Abdominal Abscesses of an African Fat-Tailed Gecko, United States, 2020
}

\author{
Ji-Yeon Hyeon ${ }^{1,2}$, Zeinab H. Helal ${ }^{1,2}{ }^{\mathbb{D}}$, Robert Polkowski ${ }^{2}$, Kristin Vyhnal ${ }^{1,2} \mathbb{D}$, Neha Mishra ${ }^{1,2}$, Junwon Kim ${ }^{1}$, \\ Guillermo R. Risatti ${ }^{1,2}$ and Dong-Hun Lee ${ }^{1, *(D)}$
}

1 Department of Pathobiology and Veterinary Science, College of Agriculture, Health and Natural Resources, University of Connecticut, Storrs, CT 06269, USA; jiyeon.hyeon@uconn.edu (J.-Y.H.); zeinab.helal@uconn.edu (Z.H.H.); Kristin.Vyhnal@uconn.edu (K.V.); neha.mishra@uconn.edu (N.M.); junwon.kim@uconn.edu (J.K.); guillermo.risatti@uconn.edu (G.R.R.)

2 Connecticut Veterinary Medical Diagnostic Laboratory, Department of Pathobiology and Veterinary Science, College of Agriculture, Health and Natural Resources, University of Connecticut, Storrs, CT 06269, USA; robert.polkowski@uconn.edu

* Correspondence: dong-hun.lee@uconn.edu; Tel.: +1-860-486-1138

\section{check for}

updates

Citation: Hyeon, J.-Y.; Helal, Z.H.; Polkowski, R.; Vyhnal, K.; Mishra, N.; Kim, J.; Risatti, G.R.; Lee, D.-H. Genomic Features of Salmonella enterica Subspecies houtenae Serotype 45:g,z51:- Isolated from Multiple Abdominal Abscesses of an African Fat-Tailed Gecko, United States, 2020. Antibiotics 2021, 10, 1322. https:// doi.org/10.3390/antibiotics10111322

Academic Editor: Nikola Puvača

Received: 8 October 2021

Accepted: 26 October 2021

Published: 29 October 2021

Publisher's Note: MDPI stays neutral with regard to jurisdictional claims in published maps and institutional affiliations.

Copyright: (c) 2021 by the authors. Licensee MDPI, Basel, Switzerland. This article is an open access article distributed under the terms and conditions of the Creative Commons Attribution (CC BY) license (https:/ / creativecommons.org/licenses/by/ $4.0 /)$.

\begin{abstract}
Salmonella enterica subsp. houtenae (S. houtenae) is a common subspecies in reptiles and has been implicated as a source of serious and life-threatening diseases in humans. Although occurrence and significance of S. houtenae infections have been extensively studied, the genetic features of $S$. houtenae have remained unknown due to a lack of available high-quality genome sequences. We obtained the complete genome sequence of S. houtenae 45:g,z51:- strain 20-369 isolated from multiple abdominal abscesses of an African fat-tailed gecko (Hemitheconyx caudicinctus) using Nanopore and Illumina sequencing technologies and generated the $4.65 \mathrm{Mbp}$ complete genome sequence of the S. houtenae str. 20-369. We annotated and analyzed the genome sequence with the aim to gain a deeper understanding of the genome characteristics associated with its pathogenicity. Overall, this study found several interesting genomic features such as pseudogene formation, virulence gene profile, and novel genomic islands. This study provides basis for an understanding possible genetic mechanism underlying pathogenicity of $S$. houtenae $45: \mathrm{g}, \mathrm{z} 51$ :- as well as a high-quality genome reference for future comparison studies.
\end{abstract}

Keywords: Salmonella enterica subspecies houtenae; reptile; African fat-tailed gecko; complete genome sequence; whole genome sequencing

\section{Introduction}

Salmonella is a genus of Gram-negative, non-sporulated and facultative anaerobe bacillus with flagella and mobility, composed of 2579 different serotypes [1]. Based on the 16S rRNA sequence and biochemical analysis, Salmonella is divided into two species: Salmonella enterica (S. enterica) and Salmonella bongori (S. bongori) [1]. S. enterica is then divided into six different subspecies, each designated with Roman numeral: enterica (I), salamae (II), arizonae (IIIa), diarizonae (IIIb), houtenae (IV) and indica (VI) [2]. Most Salmonella diseases are linked to a wide variety of serotypes of S. enterica subsp. enterica (I), being its main route of dissemination contaminated food and water [3]. However, the participation of Salmonella subsp. II-VI in cases of atypical diseases in humans, has recently been described $[4,5]$.

S. enterica subsp. houtenae (S. houtenae) was originally isolated from a cockatiel (Nymphicus hollandicus) in 1978, and 73 serotypes of S. houtenae have been described since $[6,7]$. $S$. houtenae inhabits the intestinal tract of reptiles and has been found to be prevalent in terrestrial and aquatic turtles, snakes, land Iguana, Australian sleepy lizards and captive zoo reptiles $[1,4,8]$. S. houtenae is the most prevalent subspecies identified from 31 cases of 
Salmonella infection in human with known exposure to reptiles in Germany between 2006 and 2008 [4]. S. houtenae has been reported as an opportunistic pathogen in humans, and some serovar of $S$. houtenae have been implicated as a source of serious and life-threatening diseases in humans, such as sepsis, meningitis, brain abscesses endocarditis and urinary tract infections that primarily affect children aged less than one year and immunocompromised adults $[3-5,7,9-13]$. However, the pathogenic potential of $S$. houtenae has been underestimated [14], and there is only very limited genomic information about S. houtenae. As of 8 March 2021, only six complete genome sequences of $S$. houtenae have been reported in NCBI GenBank database; S. houtenae serotypes 16:z4,z32:-, 44:z4,z23:-, and 43:z4,z23:-, and to our best knowledge, there is only two published reports of genome analysis of S. houtenae but no reports of complete genome analysis [8,14].

According to the report on laboratory-confirmed Salmonella infections during 20032013 by Centers for Disease Control and Prevention (CDC) [15], S. houtenae serotypes 50:z4,z23:-, 50:g,z51:-, 48:g,z51:-, 44:z4,z23:-, and 45:g,z51:- are the most prevalent in $S$. houtenae infection. In this study, we produced the first complete genome sequence of S. houtenae 45:g,z51:- strain 20-369 isolated from multiple abdominal abscesses of an African fat-tailed gecko in Connecticut, United States. We analyzed the genomic features of the isolate including presence of antibiotic resistance genes and chromosome mutations, pseudogenes, plasmids, virulence gene profiles, and single-nucleotide polymorphism (SNP) to establish phylogenetic relationships with other Salmonella spp.

\section{Methods}

\subsection{Bacterial Isolation and Identification}

S. houtenae str. 20-369 was isolated from multiple abdominal abscesses of a 3-year-old female African fat-tailed gecko (Hemitheconyx caudicinctus) at the Connecticut Veterinary Medical Diagnostic Laboratory (CVMDL), Department of Pathobiology and Veterinary Science, University of Connecticut in 2020. The African fat-tailed gecko had severe subacute pyogranulomatous and necrotizing oophoritis and minimal to moderate multifocal granulomatous peritonitis with intralesional bacterial colonies, and histopathology revealed infection originating in the ovary. For isolation of Salmonella spp. from the abdominal abscesses, the clinical samples were plated on brilliant green novobiocin (BGN), Xylose Lysine Tergitol-4 (XLT-4) and blood agar plates, then the plates were incubated T Dickinson, Franklin Lakes, NJ, USA) and via $16 \mathrm{~S}$ rRNA PCR amplification and a sequence alignment of the amplicon using the Basic Local Alignment Search Tool (BLAST).

\subsection{Antibiotic Susceptibility Test of S. houtenae Isolate}

The Kirby-Bauer Test disc-diffusion method was performed to determine antibiotic susceptibility of the isolate as recommended by Clinical and Laboratory Standards Institute for a consensus interpretive criterion [16]. The antibiotics tested in this study are listed in Table 1.

Table 1. Antibiotic resistance phenotype and genotype of S. houtenae str.20-369 isolate of this study.

\begin{tabular}{lll}
\hline Type & Antibiotics & Antibiotic Resistance ${ }^{*}$ \\
\hline Genotype & Aminoglycoside & aac(6 $6^{\prime}$ )-laa, \\
& Fluroquinolone & - \\
Phenotype & Gentamicin (Aminoglycosides) & $\mathrm{S}$ \\
& Streptomycin (Aminoglycosides) & $\mathrm{R}$ \\
& Amoxicillin clavulanate (B-lactam combination) & $\mathrm{S}$ \\
& Cefoxitin (Cephems) & $\mathrm{S}$ \\
& Ceftiofur (Cephems) & $\mathrm{S}$ \\
& Ceftriaxone (Cephems) & $\mathrm{S}$ \\
& Ampicillin (Penicillin) & $\mathrm{S}$ \\
& Imipenem (Carbapenems) & $\mathrm{S}$ \\
& Sulfisoxazole (Folate pathway antagonist) & $\mathrm{S}$ \\
\hline
\end{tabular}


Table 1. Cont.

\begin{tabular}{lll}
\hline Type & Antibiotics & Antibiotic Resistance * \\
\hline Trimethoprim-sulfamethoxazole (Folate & $\mathrm{S}$ \\
pathway antagonist) & $\mathrm{S}$ \\
Chloramphenicol (Phenicols) & $\mathrm{S}$ \\
Ciprofloxacin (Quinolones) & $\mathrm{S}$ \\
Enterofloxacin (Quinolones) & $\mathrm{S}$ \\
Marbofloxacin (Quinolones) & $\mathrm{S}$ \\
Nalidixic acid (Quinolones) & $\mathrm{S}$ \\
Tetracycline (Tetracyclines) & $\mathrm{S}$ \\
\hline Nitrofurantoin (Nitrofurans) &
\end{tabular}

${ }^{*}$, sensitive; $R$, resistance.

\subsection{Whole Genome Sequencing}

Two separate genomic DNA libraries were prepared according to the requirements of the Illumina and Oxford Nanopore systems. A combination of long-read Nanopore MinION and short-read Illumina MiSeq was used to generate the complete genome sequence of S. houtenae str. 20-369. For short-read sequencing, genomic DNA was extracted from pure cultures of the isolate using the DNeasy Blood and Tissue kit (Qiagen, Valencia, CA, USA) according to the manufacturer's instructions. DNA samples $(0.2 \mathrm{ng} / \mu \mathrm{L})$ were used for the library preparation using the Illumina Nextera XT DNA Library Prep Kit (Illumina, San Diego, CA, USA). The libraries were diluted to a $2 \mathrm{nM}$ concentration using the Qubit BR dsDNA HS assay kit and combined in equal volumes to form the pooled library. The library pool ( $600 \mu \mathrm{L}$ of the $10 \mathrm{pM}$ libraries) was loaded into the MiSeq reagent v2 250 cycle cartridge (Illumina, San Diego, CA, USA). The paired FASTQ files were base called from the Illumina raw sequence read data. The raw sequence reads were trimmed using Trimmomatic v0.36.6 to trim sequencing adapters, reads with a quality score $<30$ over a sliding window size of $4 \mathrm{bp}$, and reads with a sequence length $<50 \mathrm{bp}$ [17]. After trimming the adaptors and filtering low-quality reads, the clean sequence data were used for further bioinformatics analyses.

For Nanopore sequencing, genomic DNA was extracted from pure cultures of S. houtenae using the Wizard ${ }^{\circledR}$ HMW DNA Extraction Kit (Promega, Madison, WI, USA) according to the manufacturer's instructions. A MinION sequencing library was prepared using the Rapid Barcoding Sequencing Kit (SQK-RBK004; Oxford Nanopore, Oxford, UK). The library was sequenced with an R9.4.1 MinION flow cell (FlO-MIN106, Oxford Nanopore, Oxford, UK) for $48 \mathrm{~h}$ using MinKNOW v2.0 with the default settings. FAST5 files containing raw Nanopore signal data were base called and converted to FASTQ format in real-time using Guppy v3.3.0, and BBDuk v38.84 was used to trim sequences shorter with mean quality scores of less than 7 to facilitate assembly barcode and adapter sequences.

\subsection{Genome Assembly and Annotation}

The genome was assembled with Unicylcer v0.4.8. [18] providing trimmed Illumina reads as paired short reads and trimmed MinION reads as long reads. SeqSero [19] and MLST 2.0 (Multi-Locus sequence typing) [20], PlasmidFinder 2.1 [21] were used to determine Salmonella serotype, sequence type, and plasmid type, respectively. ResFinder 4.1 was used to determine the presence of acquired antimicrobial resistance genes and chromosomal mutations in the gyrA, gyrB, parC, and parE genes with settings of threshold of $90 \%$, and minimum length of $60 \%$ with raw sequencing reads [22]. Pseudofinder v0.10 (https:/ / github.com/filip-husnik/pseudofinder) (accessed on 10 March 2021), which automatically detects pseudogene candidates in prokaryotic genomes was used predict the content of pseudogenes. The assembled genome was annotated at the PATRIC annotation server with the RASTtk algorithm [23] default parameters.

SPIs were detected using BLAST against the reference sequences in previous studies [24,25] and VFDB (http:/ / www.mgc.ac.cn/cgi-bin/VFs/search.cgi, accessed on 10 March 2021) with the threshold 50\% of Grade using Geneious Prime 2020. Genomic islands 
(GI) were predicted by SIGIHMM, IslandPick, and IslandPath-DIMOB at islandviewer4 (https:/ / www.pathogenomics.sfu.ca/islandviewer, accessed on 10 March 2021).

\subsection{Phylogenetic Analysis}

A total of 30 genomes of Salmonella spp. including the complete genome of S. houtenae str. 20-369, 23 complete genomes of Salmonella spp. (S. bongori str. N268, S. enterica subsp. arizonae str. CP000880, S. enterica subsp. diarizonae str. 16SA00356, 16 serotypes of S. enterica subsp. enterica, and three serotypes of $S$. houtenae), and seven draft genomes of $S$. houtenae 45:g,z51:- were used for phylogenetic analysis. The seven S. houtenae 45:g,z51:- strains were selected based on confirmation by Seqsero and available information on source, collection year, location, Bioproject ID and Biosample ID and their FASTA-formatted contigs were downloaded from EnteroBase (http:/ / enterobase.warwick.ac.uk/species/index/senterica, accessed on 15 March 2021). The information on ten S. houtenae strains used for phylogenetic analysis is shown in Supplementary Table S1.

High-quality SNPs were identified using CSI phylogeny 1.4 and with the complete genome of $S$. houtenae str. 20-369 as a reference genome using default quality filters [26]. Maximum likelihood (ML) phylogenetic tree was constructed using RAxML-HPC v.8 with 1000 bootstrap replicates on XSEDE [27].

\subsection{Comparative Genome Analysis}

The Blast Ring Image Generator (BRIG) (v0.95) program was used to determine the genome comparison between the complete genome of S. houtenae str. 20-369 and the complete genomes of S. bongori str. N268, S. enterica subsp. arizonae str. CP000880, S. enterica subsp. diarizonae str. 16SA00356, S. enterica subsp. enterica Enteritidis (S. Enteritidis) str. P125109, Salmonella enterica subsp. enterica Typhimurium (S. Typhimurium) str. LT2, and S. houtenae CFSAN000552, 2009K170, and CVM 24399 strains (Table S1) from NCBI Reference sequence database [28]. The circular comparative genomic map was constructed by BRIG with standard default parameters and NCBI local blast-2.9.0+ suite.

\subsection{Virulence Gene Profiles}

Protein annotations associated with virulence were downloaded from the PATRIC workspace. The virulence profile of S. houtenae str. 20-369 was compared with ten $S$. houtenae strains using the virulence factor database (VFDB), a reference database for bacterial virulence factors (http:/ / www.mgc.ac.cn/cgi-bin/VFs/genus.cgi?Genus=Salmonella, accessed on 15 March 2021) (Table S1).

\section{Results and Discussion}

\subsection{Genomic Features}

The genome of $S$. houtenae str. $20-369$ has $4,651,052$ bp with a G + C content of $51.7 \%$. Annotation of the genome sequences revealed a total of 4575 putative protein-coding sequences (CDSs), 84 tRNAs, and 22 rRNAs. The isolate does not harbor any plasmids. The sequence type was ST107 by multi-locus sequence typing (MLST) analysis which is the most frequent sequence type in S. houtenae 45:g,z51:- in the Enterobase.

The S. houtenae str. 20-369 was assigned to serotype IV 45:g,z51:- or IIIa 45:g,z51:- using the SeqSero tool (Salmonella Serotyping by Whole Genome Sequencing). In the maximum likelihood (ML) phylogeny, the S. houtenae str. 20-369 clustered with other S. houtenae strains, and the $S$. houtenae strains formed a well-supported monophyletic clade with high bootstrap value (Figure 1a). In a subtree (Figure 1b), the $S$. houtenae str. 20-369 clustered with the seven S. houtenae 45:g,z51:- strains with $>97 \%$ sequence identity. 


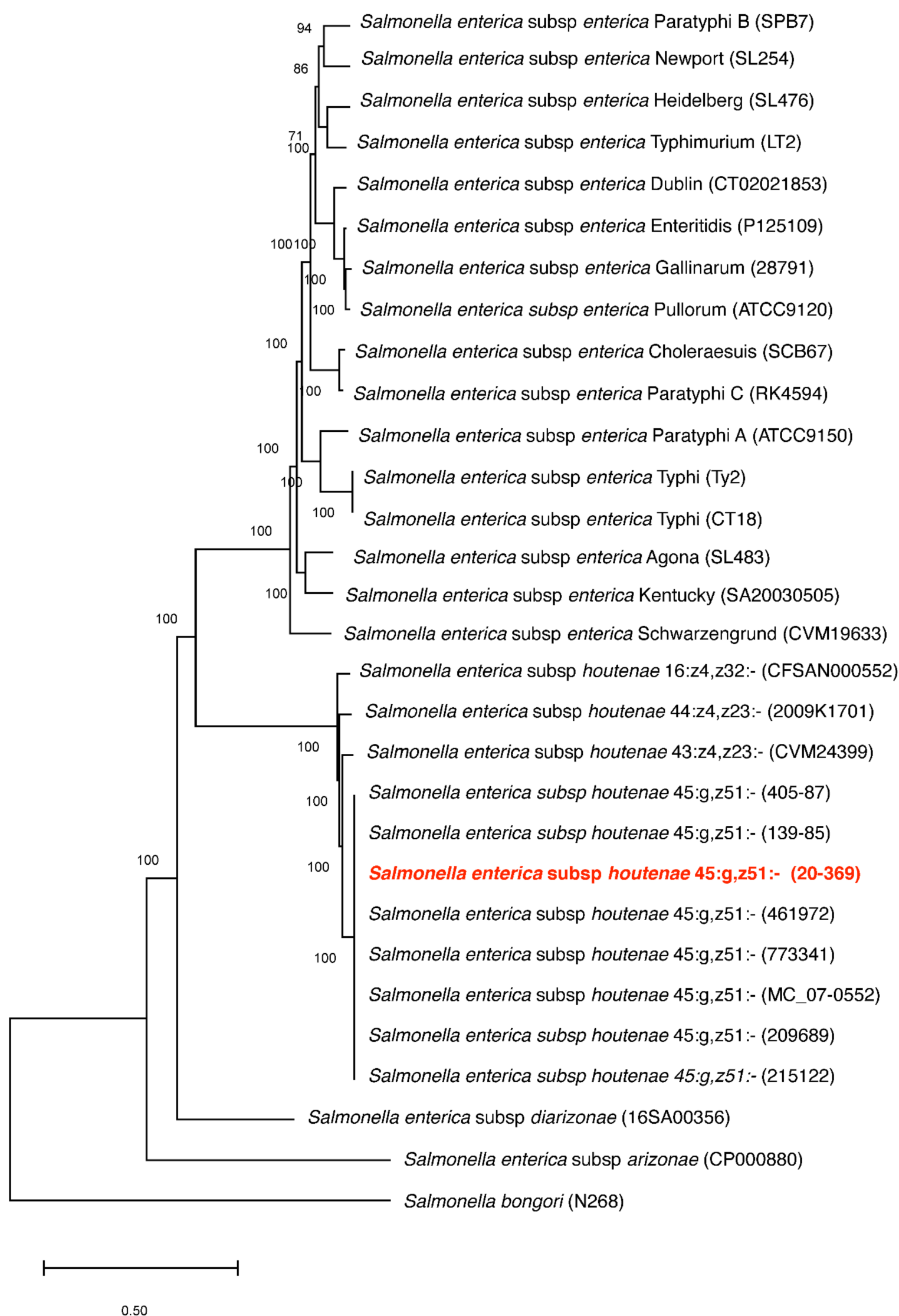

(a)

Figure 1. Cont. 


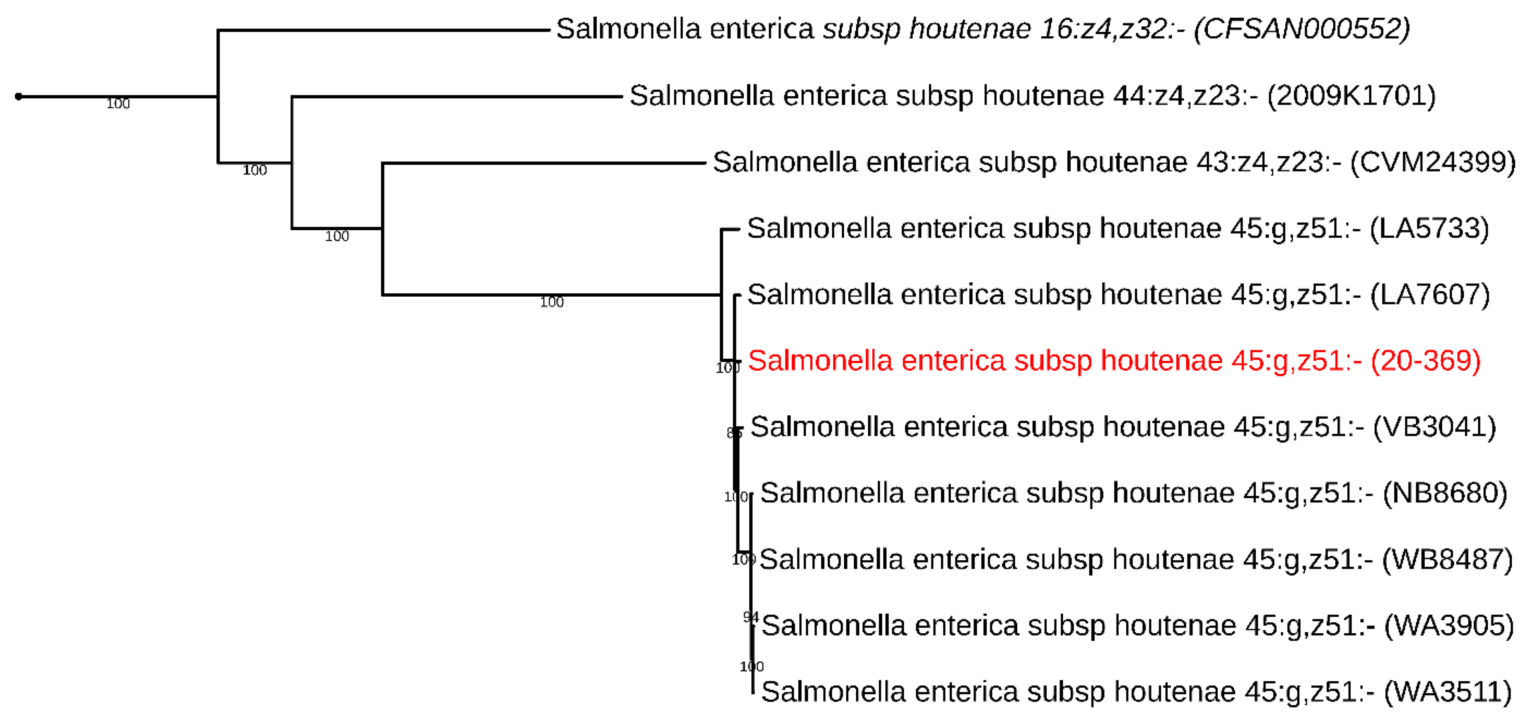

(b)

Figure 1. Phylogenetic analysis of 30 complete genomes of Salmonella spp., including strain 20-369, using SNP analysis. (a) The phylogeny was rooted at midpoint. (b) The phylogeny of Salmonella enterica subsp. houtenae strains. The scale bars show the number of substitutions per site. The numerical values represent 1000 bootstrap replicate values above 0.9 .

The percentage of detected pseudogene candidates was 5.75\% (244 of 4241). For Salmonella, pseudogene accumulation has been regarded as a signature of host-specific pathogenic bacteria (S. Dublin with 289 pseudogenes) as compared to their host-generalist relatives (S. Enteritidis with 111 pseudogenes) [29]. When comparing the percentage of pseudogenes normalized to the total ORFs, our isolate contains $5.7 \%$ of pseudogenes. Similarly, $S$. Dublin which is bovine-adapted have a pseudogene content of about $5.7 \%$, and other narrow-host-range Salmonella serovars like S. Choleraesuis (pigs), S. Typhi (humans), S. Gallinarum, and Pullorum (birds) showed a higher percentage of Pseudogenes $(6.5-7.6 \%)$ in other study [30]. Therefore, available data and our results further support host-adaptation of $S$. houtenae to reptile.

\subsection{Antibiotic Resistance}

S. houtenae str. 20-369 was susceptible to all antibiotics tested except streptomycin (aminoglycosides) and carries the aac $\left(6^{\prime}\right)$-Iaa gene which is a chromosomal-encoded aminoglycoside $6^{\prime} \mathrm{N}$-acetyltransferase and a point mutation T57S in the $\operatorname{par} \mathrm{C}$ gene, but mutations were not observed in gyrA, gyrB, or parE genes.

In relation to the antimicrobial susceptibility of Salmonella, there are few data concerning the antimicrobial resistance of $S$. houtenae compared with $S$. enterica subspecies enterica. It has been observed that $S$. houtenae strain isolated from reptiles have resistance to antimicrobials, two $S$. houtenae isolates from gecko resistant to ampicillin or tetracycline [31] and S. houtenae 45:g,z51:- isolated from healthy captive bred female veiled chameleons resistant to streptomycin [32]. In the previous study of the whole genome analysis of S. houtenae [14], the isolate shows the susceptible phenotype to all antimicrobials tested but carries the antimicrobial resistance gene associated with aminoglycoside resistance (Aac6-Iaa_AGly), which it could be under-expressed. In our study, phenotypic antimicrobial resistance was concordant with genotypic antimicrobial resistance for streptomycin but 
not for quinolone. It has been reported that the mutations in the parC are not essential for quinolone resistance, but it can result in a high level of fluoroquinolone resistance [33].

\subsection{Genomic Islands (GI) and Salmonella Pathogenicity Islands (SPI)}

A total of 46 GIs, designated as GI-1 to GI-46, were predicted using SIGIHMM, IslandPick and IslandPath-DIMOB (Figure 2, Table S2). By comparing the sequence-based similarity of the genomes using an all-against-all BLAST comparison, we identified six novel islands (GI-19, GI-21, GI-22, GI-24, GI-36, and GI-46) observed only in the complete genomes of $S$. houtenae strains (Figure 2). Detailed inspection of these islands revealed the presence of predicted proteins related to antitoxin HigA, toxin $\mathrm{HigB}$, fimbrial proteins, transposase, phage-related protein, error-prone repair protein, oxidoreductase, transcriptional regulator (Lys family, GntR family), Type VI secretion system (T6SS), and phosphotransferase (PTS) system (Table S2). In addition, specific portions of seven novel genomic islands (GI-6, GI-14, GI-15, GI-16, GI-18, GI-30, and GI-32) were found in S. houtenae str. 20-369 but absent in the rest of the genomes analyzed (Figure 2). These novel genomic islands contain the genes encoding the proteins associated with type III secretion system (TTSS), antitoxin HigA, toxin HigB, transposase, sugar transferase, error-prone repair protein, isochorismatase family protein, and hypothetical proteins (Table S2).

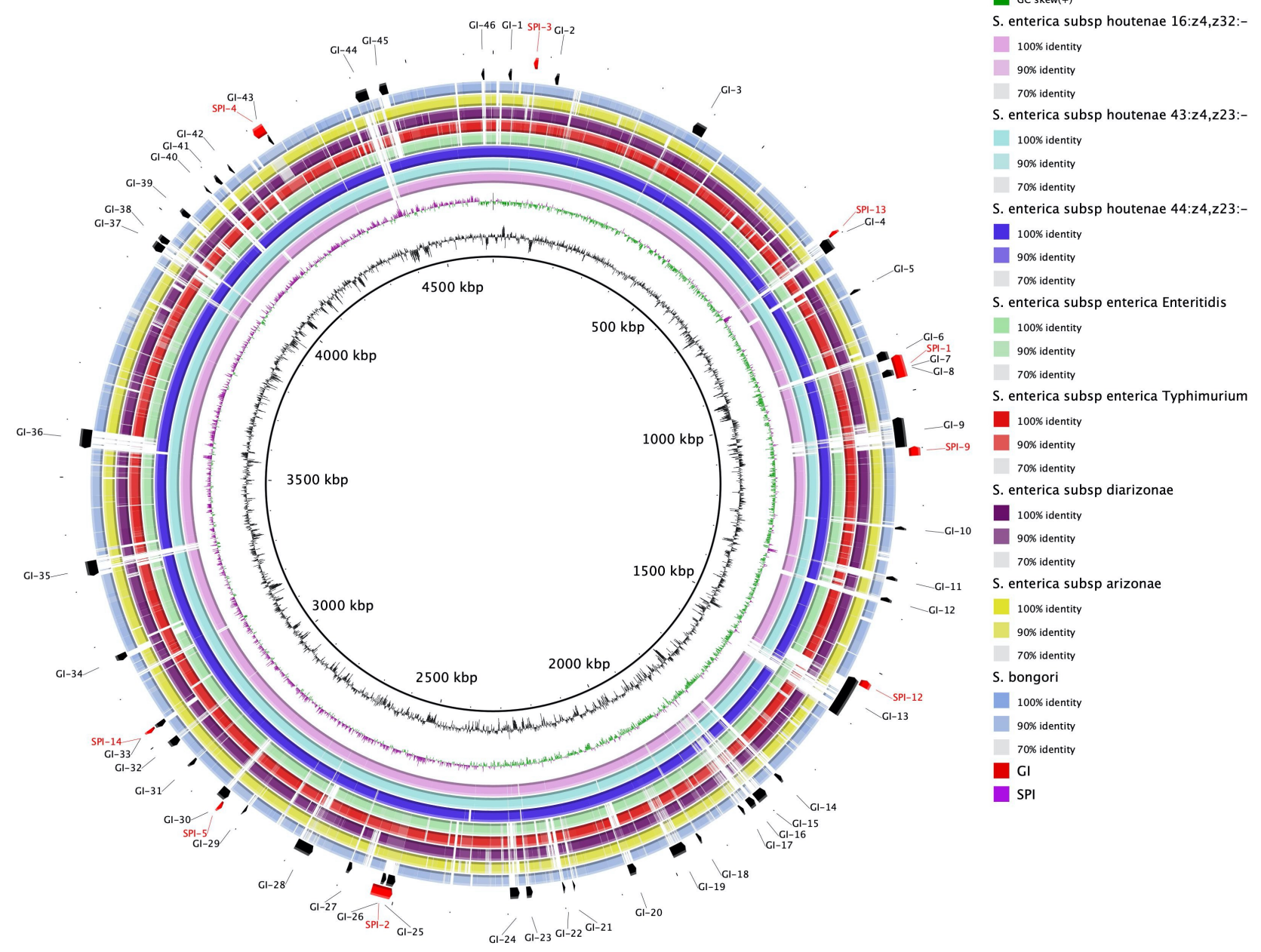

Figure 2. Blast Ring Image Generator (BRIG) diagram showing the complete genomes of Salmonella spp. strains, with genome of strain 20-369 as a reference. The outer circle contains genomic island regions (black) and Salmonella Pathogenicity Island (SPI) (red) of strain 20-369. GC content is also shown in the figure. 
In a previous study [14], S. houtenae str. CFSAN039533 shared 234 (86\%) of selected proteins with $S$. Enteritidis str. 77-1427 and $S$. Typhimurium str. LT2, but three genes related to virulence functions $(C l f A)$ and regulation systems ( $H i g A, Y g f l)$ were classified as specific for CFSAN039533 strain. In our study, the genomic islands contain the genes encoding the HigB/HigA toxin/antitoxin system which is one of the toxin-antitoxin (TA) systems, which are prevalent in most bacterial and archaeal genomes, and one of the emerging physiological roles of TA systems is to help regulate pathogenicity [34]. Genes for the HigB/HigA TA systemare found in the chromosomes of the many pathogens such as Vibrio cholera, Streptococcus pneumoniae, Acinetobacter baumannii, Yersinia pestis, E. coli CFT073 and E. coli O157:H7 [34], but not reported in Salmonella spp. [35,36].

BLAST results showed that all three isolates harbor major SPIs, including SPI-1 to SPI-5, SPI-9, SPI-12 to SPI-14 (Figure 2). The SPI-1 to SPI-5 has been described as main SPIs of Salmonella genome [37], and many studies have reported that SPI-1, SPI-2 and SPI-4 are conserved genetic islands, while SPI-3 and SPI-5 displayed a variable genetic information [14]. In the previous study [14], the CFSAN039533 strain displayed virulence genes belonging to SPI-1, SPI-2, SPI-3 and SPI-5 when they performed a virulence gene database constituted with representative genes of SPIs (SPI-1 to SPI-5 and SPI-7), but the strain did not carry siiD and siiE of SPI-4. However, in this study all S. houtenae strains carry siiD but none of them carries siiF (data not shown). All S. houtenae 45:g,z51:- strains except the 20-369 and MC_07-0552 strains carry siiE (data not shown).

\subsection{Virulence Gene Profile}

Genome sequences of $11 \mathrm{~S}$. houtenae strains including our isolate were analyzed for virulence genes using the VFDB (Figure 3). The $S$. houtenae strains carry a conserved virulence gene profile in fimbrial adherence determinants (csg, fim, and $\operatorname{sth} A)$, magnesium uptake genes ( $m g t B$ and $m g t C)$, regulation ( $p h o P$ and $p h o Q)$, TTSS (SPI-1 and SPI-2 encode), and stress adaptation $(\operatorname{sod} C l)$ but show various profiles in TTSS effectors translocated via both systems (slrp), TTSS-1 translocated effectors (sopA), TTSS-2 translocated effectors (sseF, sseI/ssaB, sse), sifA, sopD2, gog, B sseK1, spvC, and spvD) and toxin (spvB). Only the S. houtenae 16:z4,z32:- str. CFSAN000552 carries spvB, spvC, and spvD.

Eight $S$. houtenae 45:g,z51:- showed identical virulence gene profile except in five virulence genes; slrp, sop A, sseF, sseI/ssaB and $g \circ g B$. Compared to other S. houtenae isolates, $S$. houtenae str. 20-369 does not carry seven virulence genes associated TTSS translocated effectors (sopA, sseI/ssaB, sopD2, gogB, sseK1, spvC, and spvD) and toxin gene spvB (Figure 3). The $S$. houtenae str. 20-369 has an identical virulence gene profile with $S$. houtenae 45:g,z51:str. 461972, which was isolated from human in United Kingdom in 2006 (Figure 3 and Table S1), of which supports that $S$. houtenae is an opportunistic pathogen.

It is notable that the cases found for $S$. houtenae infections have been focused on the brain, and it appears that this subspecies has an affinity for the brain, causing extraintestinal infections [1]. However, due to a lack of available complete genome sequences of $S$. houtenae, the genome comparison study for genetic relatedness and pathogenic mechanism could not be performed in this study.

In this study, we announced the first complete and closed genome sequence of S. houtenae 45:g,z51:- isolated from a reptile in United States and analyzed the antibiotic resistance and virulence gene profiles. Overall, this study found a number of interesting genomic features such as pseudogene formation and novel genomic islands. With this study, we provide an importance basis for an understanding of the genetic mechanism that underlies pathogenicity $S$. houtenae $45: \mathrm{g}$,z51:-, as well as a high-quality reference for future genome comparison studies. 

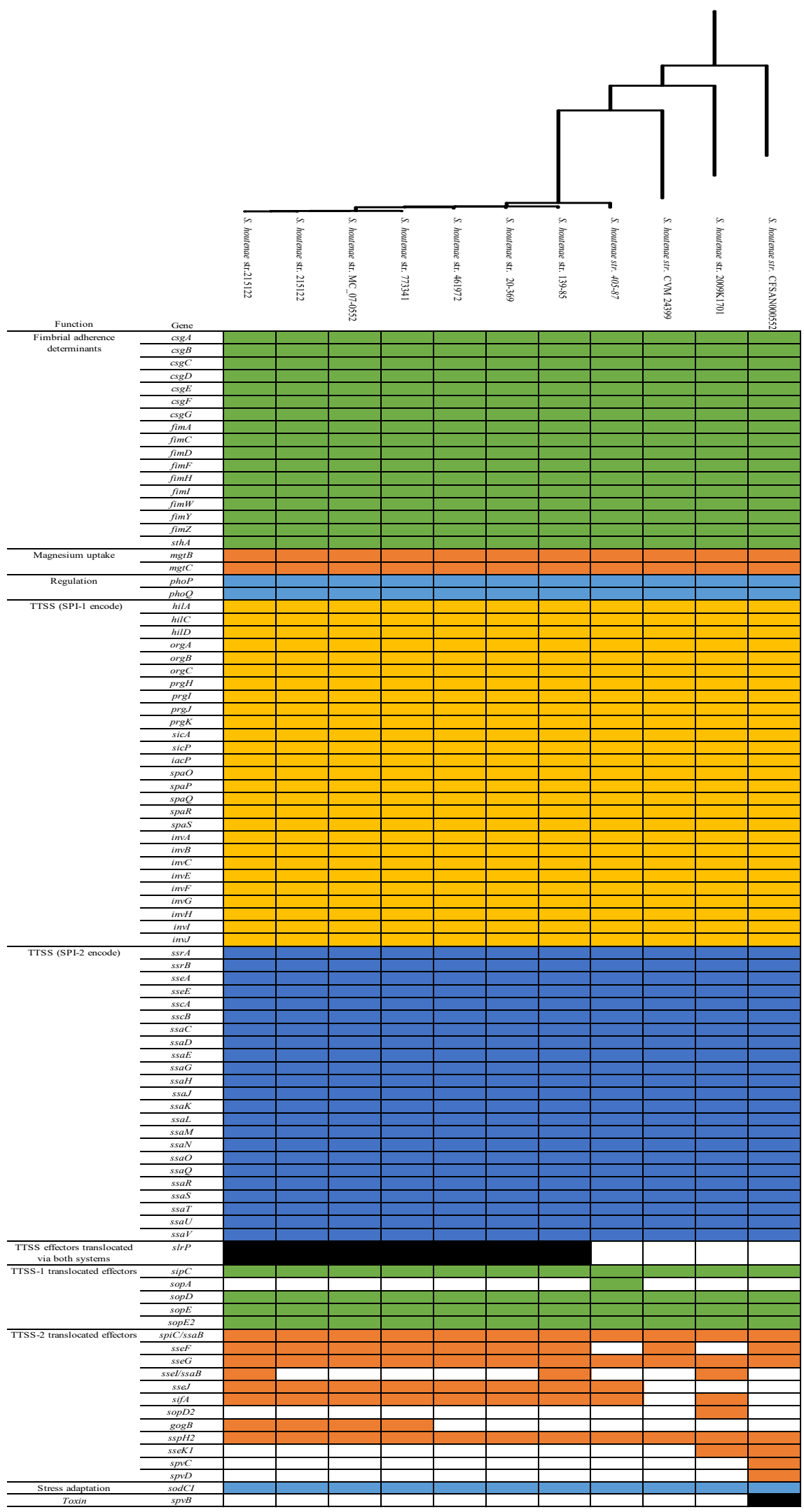

Figure 3. Virulence gene profile of 11 Salmonella enterica subsp. houtenae strains including strain 20-369. 
Supplementary Materials: The following are available online at https://www.mdpi.com/article/10 .3390 /antibiotics10111322/s1, Table S1. List of Salmonella enterica subsp. houtenae strains downloaded as FASTA-formatted contigs from NCBI and EnteroBase analyzed in this study $(n=10)$, Table S2. The genomic islands (GI) and annotation of the strain 20-369.

Author Contributions: Conceptualization, D.-H.L. and G.R.R.; microbiology, Z.H.H. and R.P.; pathology: K.V. and N.M.; genome sequencing: J.-Y.H., J.K. and D.-H.L.; bioinformatics: J.-Y.H., J.K. and D.-H.L.; writing—original draft preparation, J.-Y.H.; writing—review and editing, D.-H.L. and G.R.R. All authors have read and agreed to the published version of the manuscript.

Funding: This work was partially supported by projects University of Connecticut SPS \#180229 and \#181033.

Data Availability Statement: The complete genome sequence of the isolate 20-369 in this study have been deposited in the National Center for Biotechnology Information (NCBI)'s under the Bioproject accession number PRJNA725952.

Acknowledgments: We thank the unconditional support of staff and faculty from the Connecticut Veterinary Medical Diagnostic Laboratory (CVMDL), Department of Pathobiology and Veterinary Science, CAHNR, University of Connecticut.

Conflicts of Interest: The authors declare that they have no competing interest.

\section{References}

1. Lamas, A.; Miranda, J.M.; Regal, P.; Vazquez, B.; Franco, C.M.; Cepeda, A. A comprehensive review of non-enterica subspecies of Salmonella enterica. Microbiol. Res. 2018, 206, 60-73. [CrossRef] [PubMed]

2. Su, L.H.; Chiu, C.H. Salmonella: Clinical importance and evolution of nomenclature. Chang. Gung. Med. J. 2007, 30, 210-219. [PubMed]

3. Chen, H.M.; Wang, Y.; Su, L.H.; Chiu, C.H. Nontyphoid Salmonella infection: Microbiology, clinical features, and antimicrobial therapy. Pediatr. Neonatol. 2013, 54, 147-152. [CrossRef] [PubMed]

4. Editorial Team; Bertrand, S.; Rimhanen-Finne, R.; Weill, F.X.; Rabsch, W.; Thornton, L.; Perevoščikovs, J.; van Pelt, W.; Heck, M. Salmonella infections associated with reptiles: The current situation in Europe. Eurosurveillance 2008, 13, 18902. [CrossRef]

5. Abbott, S.L.; Ni, F.C.; Janda, J.M. Increase in extraintestinal infections caused by Salmonella enterica subspecies II-IV. Emerg. Infect. Dis. 2012, 18, 637-639. [CrossRef]

6. Phillips, W.E., Jr.; Hatkin, J.M. Isolation of Salmonella houtenae from a cockateel. Avian Dis. 1978, 22, 350-353. [CrossRef]

7. Tabarani, C.M.; Bennett, N.J.; Kiska, D.L.; Riddell, S.W.; Botash, A.S.; Domachowske, J.B. Empyema of preexisting subdural hemorrhage caused by a rare Salmonella species after exposure to bearded dragons in a foster home. J. Pediatr. 2010, 156, 322-323. [CrossRef]

8. Zhu, S.; Wang, H.L.; Wang, C.; Tang, L.; Wang, X.; Yu, K.J.; Liu, S.L. Non-contiguous finished genome sequence and description of Salmonella enterica subsp. houtenae str. RKS3027. Stand. Genom. Sci. 2013, 8, 198-205. [CrossRef]

9. Krath, M.L.; Hillhouse, A.E.; Little, S.V.; Lawhon, S.D. MiSeq Sequencing of Salmonella enterica subsp. houtenae Isolates from a Dog Treated for Hind-Limb Paresis. Microbiol. Resour. Announc. 2020, 9, e00655-20. [CrossRef]

10. Lourenco, M.C.; dos Reis, E.F.; Valls, R.; Asensi, M.D.; Hofer, E. Salmonella enterica subsp houtenae serogroup O:16 in a HIV positive patient: Case report. Rev. Inst. Med. Trop. Sao Paulo 2004, 46, 169-170. [CrossRef]

11. Ma, J.S.; Chen, P.Y.; Lau, Y.J.; Chi, C.S. Brain abscess caused by Salmonella enterica subspecies houtenae in a patient with chronic granulomatous disease. J. Microbiol. Immunol. Infect. 2003, 36, 282-284.

12. Nimir, A.R.; Ibrahim, R.; Ibrahim, I.A. Salmonella meningitis in a paediatric patient caused by Salmonella enterica serotype Houtenae. BMJ Case Rep. 2011, 2011, bcr0420114096. [CrossRef]

13. Wybo, I.; Potters, D.; Plaskie, K.; Covens, L.; Collard, J.M.; Lauwers, S. Salmonella enterica subspecies houtenae serotype 44:z4, z23:-as a rare cause of meningitis. Acta Clin. Belg. 2004, 59, 232-234.

14. Castaneda-Ruelas, G.M.; Burgeno-Roman, A.; Jimenez-Edeza, M. Genetics and physiology of Salmonella houtenae isolated from a river in Mexico provides insight into the aquatic habitat influence on its adaptation and pathogenesis. Infect. Genet. Evol. 2020, 83, 104326. [CrossRef] [PubMed]

15. Centers for Disease Control and Prevention. National Enteric Disease Surveillance: Salmonella Annual Report, 2013; Centers for Disease Control and Prevention: Atlanta, GA, USA, 2016.

16. Clinical and Laboratory Standards Institute. VET 08 Performance Standards for Antimicrobial Disk and Dilution Susceptibility Tests for Bacteria Isolated From Animals; Clinical and Laboratory Standards Institute: Wayne, PA, USA, 2018.

17. Bolger, A.M.; Lohse, M.; Usadel, B. Trimmomatic: A flexible trimmer for Illumina sequence data. Bioinformatics 2014, 30, 2114-2120. [CrossRef] [PubMed]

18. Wick, R.R.; Judd, L.M.; Gorrie, C.L.; Holt, K.E. Unicycler: Resolving bacterial genome assemblies from short and long sequencing reads. PLoS Comput. Biol. 2017, 13, e1005595. [CrossRef] [PubMed] 
19. Zhang, S.; den Bakker, H.C.; Li, S.; Chen, J.; Dinsmore, B.A.; Lane, C.; Lauer, A.C.; Fields, P.I.; Deng, X. SeqSero2: Rapid and Improved Salmonella Serotype Determination Using Whole-Genome Sequencing Data. Appl. Environ. Microbiol. 2019, 85, e01746-19. [CrossRef] [PubMed]

20. Larsen, M.V.; Cosentino, S.; Rasmussen, S.; Friis, C.; Hasman, H.; Marvig, R.L.; Jelsbak, L.; Sicheritz-Ponten, T.; Ussery, D.W.; Aarestrup, F.M.; et al. Multilocus sequence typing of total-genome-sequenced bacteria. J. Clin. Microbiol. 2012, 50, 1355-1361. [CrossRef] [PubMed]

21. Carattoli, A.; Zankari, E.; Garcia-Fernandez, A.; Voldby Larsen, M.; Lund, O.; Villa, L.; Moller Aarestrup, F.; Hasman, H. In silico detection and typing of plasmids using PlasmidFinder and plasmid multilocus sequence typing. Antimicrob. Agents Chemother. 2014, 58, 3895-3903. [CrossRef]

22. Zankari, E.; Hasman, H.; Cosentino, S.; Vestergaard, M.; Rasmussen, S.; Lund, O.; Aarestrup, F.M.; Larsen, M.V. Identification of acquired antimicrobial resistance genes. J. Antimicrob. Chemother. 2012, 67, 2640-2644. [CrossRef]

23. Brettin, T.; Davis, J.J.; Disz, T.; Edwards, R.A.; Gerdes, S.; Olsen, G.J.; Olson, R.; Overbeek, R.; Parrello, B.; Pusch, G.D.; et al. RASTtk: A modular and extensible implementation of the RAST algorithm for building custom annotation pipelines and annotating batches of genomes. Sci. Rep. 2015, 5, 8365. [CrossRef]

24. Gerlach, R.G.; Walter, S.; McClelland, M.; Schmidt, C.; Steglich, M.; Prager, R.; Bender, J.K.; Fuchs, S.; Schoerner, C.; Rabsch, W.; et al. Comparative whole genome analysis of three consecutive Salmonella diarizonae isolates. Int. J. Med. Microbiol. 2017, 307, 542-551. [CrossRef]

25. Suez, J.; Porwollik, S.; Dagan, A.; Marzel, A.; Schorr, Y.I.; Desai, P.T.; Agmon, V.; McClelland, M.; Rahav, G.; Gal-Mor, O. Virulence gene profiling and pathogenicity characterization of non-typhoidal Salmonella accounted for invasive disease in humans. PLoS ONE 2013, 8, e58449. [CrossRef] [PubMed]

26. Kaas, R.S.; Leekitcharoenphon, P.; Aarestrup, F.M.; Lund, O. Solving the problem of comparing whole bacterial genomes across different sequencing platforms. PLOS ONE 2014, 9, e104984. [CrossRef] [PubMed]

27. Stamatakis, A. RAxML version 8: A tool for phylogenetic analysis and post-analysis of large phylogenies. Bioinformatics 2014, 30, 1312-1313. [CrossRef] [PubMed]

28. Alikhan, N.F.; Petty, N.K.; Ben Zakour, N.L.; Beatson, S.A. BLAST Ring Image Generator (BRIG): Simple prokaryote genome comparisons. BMC Genom. 2011, 12, 402. [CrossRef]

29. Betancor, L.; Yim, L.; Martinez, A.; Fookes, M.; Sasias, S.; Schelotto, F.; Thomson, N.; Maskell, D.; Chabalgoity, J.A. Genomic Comparison of the Closely Related Salmonella enterica Serovars Enteritidis and Dublin. Open Microbiol. J. 2012, 6, 5-13. [CrossRef]

30. Uelze, L.; Borowiak, M.; Deneke, C.; Jacobs, C.; Szabo, I.; Tausch, S.H.; Malorny, B. First complete genome sequence and comparative analysis of Salmonella enterica subsp. diarizonae serovar 61:k:1,5,(7) indicates host adaptation traits to sheep. Gut. Pathog. 2019, 11, 48. [CrossRef]

31. Singh, B.R.; Singh, V.; Ebibeni, N.; Singh, R.K. Antimicrobial and Herbal Drug Resistance in Enteric Bacteria Isolated from Faecal Droppings of Common House Lizard/Gecko (Hemidactylus frenatus). Int. J. Microbiol. 2013, 2013, 340848. [CrossRef]

32. Romero, S.B.; Knotek, Z.; Čížek, A.; Masaříková, M.; Myšková, P. The incidence and antibiotic resistance of Salmonella species isolated from cloacae of captive veiled chameleons. Acta Vet. Brno. 2015, 84, 209-213. [CrossRef]

33. Hooper, D.C.; Jacoby, G.A. Topoisomerase Inhibitors: Fluoroquinolone Mechanisms of Action and Resistance. Cold Spring Harb. Perspect. Med. 2016, 6, a025320. [CrossRef] [PubMed]

34. Wood, T.L.; Wood, T.K. The HigB/HigA toxin/antitoxin system of Pseudomonas aeruginosa influences the virulence factors pyochelin, pyocyanin, and biofilm formation. Microbiologyopen 2016, 5, 499-511. [CrossRef] [PubMed]

35. De la Cruz, M.A.; Zhao, W.; Farenc, C.; Gimenez, G.; Raoult, D.; Cambillau, C.; Gorvel, J.P.; Meresse, S. A toxin-antitoxin module of Salmonella promotes virulence in mice. PLoS Pathog. 2013, 9, e1003827. [CrossRef]

36. The PLOS Pathogens Editors. Retraction: A Toxin-Antitoxin Module of Salmonella Promotes Virulence in Mice. PLoS Pathog. 2020, 16, e1009031. [CrossRef]

37. Rychlik, I.; Karasova, D.; Sebkova, A.; Volf, J.; Sisak, F.; Havlickova, H.; Kummer, V.; Imre, A.; Szmolka, A.; Nagy, B. Virulence potential of five major pathogenicity islands (SPI-1 to SPI-5) of Salmonella enterica serovar Enteritidis for chickens. BMC Microbiol. 2009, 9, 268. [CrossRef] 